\title{
Early gas stripping as the origin of the darkest galaxies in the Universe
}

\author{
L. Mayer ${ }^{1,2}$, S. Kazantzidis ${ }^{3,4}$, C. Mastropietro ${ }^{5}$, \& J. Wadsley ${ }^{6}$ \\ ${ }^{1}$ Institut für Astronomie, ETH Zürich, Wolfgang-Pauli-Strasse 16 , CH-8093 Zürich, Switzerland. \\ ${ }^{2}$ Institute for Theoretical Physics, University of Zurich, Winterthurestrasse 190, CH-8057 Zürich. \\ ${ }^{3}$ Kavli Institute for Particle Astrophysics and Cosmology, Department of Physics, Stanford University, P.O. \\ Box 20450, MS 29, Stanford, CA 94309 USA. \\ ${ }^{4}$ Kavli Institute for Cosmological Physics, Department of Astronomy \& Astrophysics, The University of \\ Chicago, Chicago, IL 60637 USA. \\ ${ }^{5}$ Universitäts Sternwarte München, Scheinerstrasse 1, D-81679 München, Germany. \\ ${ }^{6}$ Department of Physics and Astronomy, McMaster University, Hamilton, ON L8S 4M1, Canada.
}

The known galaxies most dominated by dark matter (Draco, Ursa Minor and Andromeda IX) are satellites of the Milky Way and the Andromeda galaxies ${ }^{1-4}$. They are members of a class of faint galaxies, devoid of gas, known as dwarf spheroidals $^{3-5}$, and have by far the highest ratio of dark to luminous matter ${ }^{3,6}$. None of the models proposed to unravel their origin $^{7-10}$ can simultaneously explain their exceptional dark matter content and their proximity to a much larger galaxy. Here we report simulations showing that the progenitors of these galaxies were probably gas-dominated dwarf galaxies that became satellites of a larger galaxy earlier than the other dwarf spheroidals. We find that a combination of tidal shocks and ram pressure swept away the entire gas content of such progenitors about ten billion years ago because heating by the cosmic ultraviolet background kept the gas loosely bound: a tiny stellar component embedded in a relatively massive dark halo survived until today. All luminous galaxies should be surrounded by a few extremely dark-matter-dominated dwarf spheroidal satellites, and these should have the shortest orbital periods among dwarf spheroidals because they were accreted early.

Draco, Ursa Minor and Andromeda IX have mass-to-light ratios $(M / L)$ larger than 100, but the majority of the other dwarf spheroidals (dSphs) in the Local Group have a lower $M / L$, of order $10-30^{1}$, typical among dwarf galaxies ${ }^{11,12}$. Another important difference is that Draco and Ursa Minor nearly stopped forming stars more than 10 billion years ago, while other dSphs continued to form stars for many billions of years ${ }^{2}$. The modest 
potential well of these extreme dwarfs cannot be the one property that determined their nature. Their halo masses are too large to invoke suppression of gas accretion owing to the cosmic ultraviolet background at high redshift ${ }^{9,13}$ or blow-out due to supernovae winds ${ }^{14}$. Tidal shocks occurring as a dwarf repeatedly approaches the primary galaxy can transform rotationally supported systems resembling dwarf irregular galaxies (dIrrs) into systems dominated by random motions, similar to dSphs ${ }^{10}$. This tidal stirring can explain why dSphs are more clustered around the primary galaxies relative to dIrrs but it leaves a significant gas component inside the dwarf, so that star formation can continue for several billions of years instead of being truncated early. Ram pressure in a hot gaseous corona could strip their gas completely ${ }^{7,15}$ but the limitations of simulations so far have not allowed for firm predictions. For instance, existing calculations keep the structure of the stars and halo fixed in time and neglect radiative cooling and heating of the gas ${ }^{16,17}$.

These earlier studies have explored the effect of a single gas removal mechanism and are, at best, only qualitatively consistent with the current structure formation paradigm, a model with cold dark matter and a cosmological constant $(\Lambda \mathrm{CDM})$. Recent attempts to study the evolution of dwarf satellites directly in cosmological simulations rely on semi-analytical methods to model the baryonic component rather than solving the fluid equations ${ }^{18}$. These models neglect ram pressure, and since stripping by tides is slow and inefficient, they cannot explain the complete absence of gas and early truncation of star formation of the darkest dSphs.

In cold dark matter models the present-day spatial distribution of subhalos within primary halos retains some memory of their infall time ${ }^{19}$. Satellites orbiting closer to the primaries were on average accreted earlier than those orbiting at larger distances. Interestingly, Draco and Ursa Minor lie at 68 and $86 \mathrm{kpc}$, respectively from the Milky Way, Andromeda IX at $45 \mathrm{kpc}$ from Andromeda, while other dSphs orbit as far as $200 \mathrm{kpc}$ from the primaries ${ }^{1}$. We use a high resolution $\Lambda$ CDM dark matter-only cosmological simulation of the formation of a Milky Way-sized halo ${ }^{20}$ (see also Supplementary Information). At $z=0$ we identify three subhalos having distances below $100 \mathrm{kpc}$ from the center and with a peak circular velocity in the range $25-30 \mathrm{~km} / \mathrm{s}^{6}$ (Figure 1 ). We track the orbits of the satellites back in time and find that two of them were accreted early, between $z=2.5$ and $z=1.5$.

We then construct a high resolution N-body + smoothed particle hydrodynamics (SPH) model of a dwarf galaxy satellite having a disk of stars and gas inside a cold dark matter halo (Figure 1) with a peak velocity of about $40 \mathrm{~km} / \mathrm{s}$, comparable to that of the two identified cosmological subhalos before they were accreted onto the Milky Way (see Supplementary Information). We assume that $80 \%$ of the baryonic disk mass is in a gas component. The inefficient conversion of gas into stars is expected at these low mass scales since most of the gas will have densities below the threshold for star formation ${ }^{21}$. In addition, at $z>2$ the gas in the dwarf is heated to a temperature of over $10^{4} \mathrm{~K}$ and ionized by the cosmic UV radiation, which further suppresses star formation.

The dwarf model is placed on an eccentric orbit inside a massive Milky Way-sized halo 


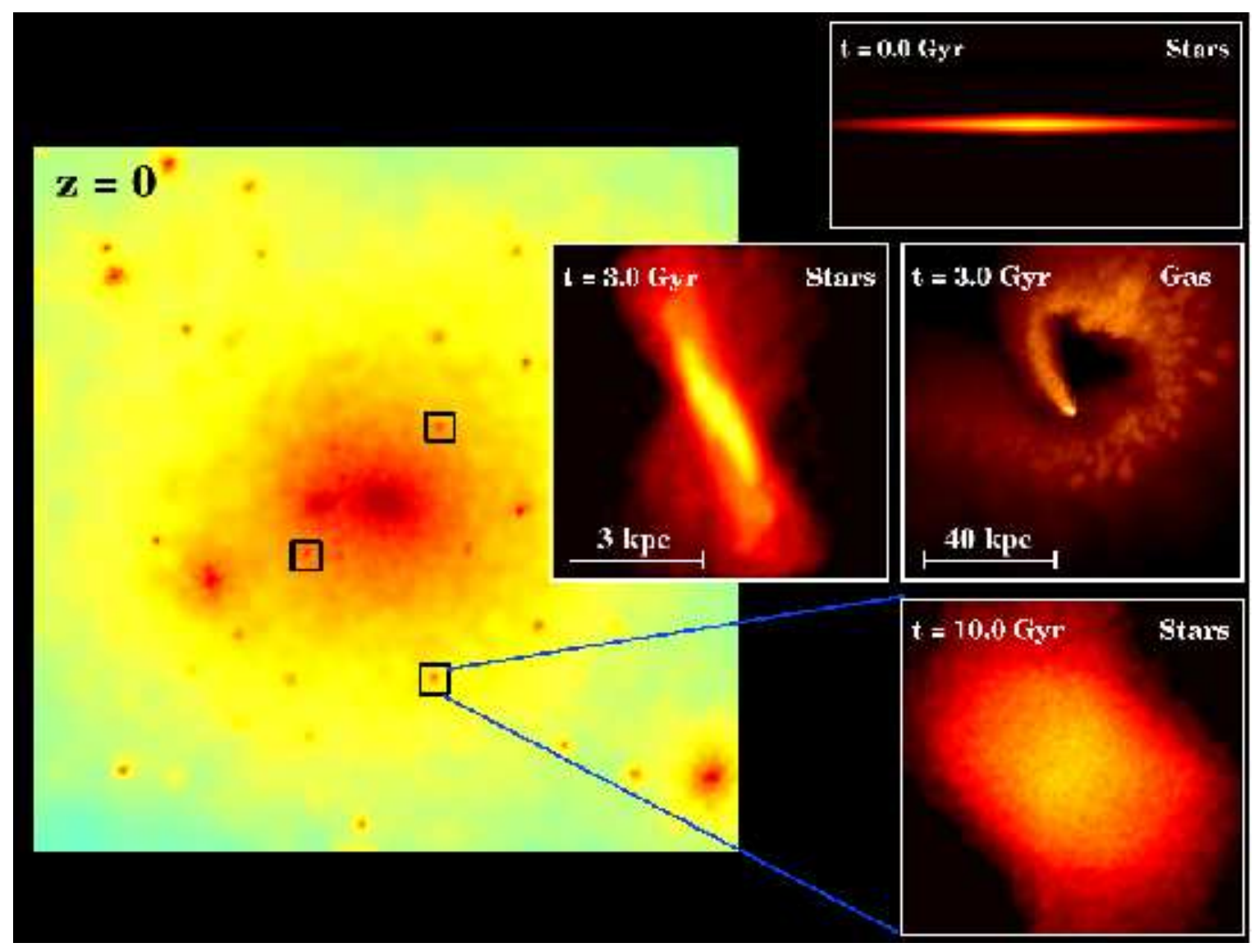

Figure 1. Morphological evolution of the dwarf satellite galaxy. Left: Color-coded projected density map of the cosmological run at $z=0$; the box is $260 \mathrm{kpc}$ on a side, which corresponds to the virial radius of the Milky Way-sized halo. The peak density along the line of sight is shown, ranging from $10^{-29}-10^{-24} \mathrm{~g} / \mathrm{cm}^{3}$, with the color coding from blue (lowest density), through yellow, then red, to brown (highest density). The three satellites that meet the distance and circular velocity constraints (see text) are highlighted with black boxes. Right: From top to bottom the insets show the stellar component of the dwarf galaxy, color coded in projected density, at different times. Only regions with densities in the range $10^{-28}-10^{-23} \mathrm{~g} / \mathrm{cm}^{3}$ are shown, with the color coding from dark red (lowest density) to yellow (highest densities). In the top inset, the initial disk is shown edge-on (the box is $8 \mathrm{kpc}$ on a side). In the left middle inset, the system is close to the second pericenter passage; the stars have assumed a strong bar-like configuration and heating is evident in the outskirts (the box is $7 \mathrm{kpc}$ on a side). In the bottom inset, the end state is shown; the bar has been heated into a diffuse spheroid and any disk-like feature has been erased (the box is $4 \mathrm{kpc}$ on a side and a a projection along a random line of sight is shown). In the right middle inset, the trail of gas produced by ram pressure is shown, while even the residual gas in the center is stripped. The color-coded gas density projected onto the orbital plane is shown (densities in the range $10^{-30}-10^{-23} \mathrm{~g} / \mathrm{cm}^{3}$, color coding as above), and the box is $100 \mathrm{kpc}$ on a side. 
Mayer et al.

model which is a replica of that in the cosmological simulation. We include radiative cooling as well as the heating and ionizing flux from the cosmic ultraviolet background radiation $^{22}$, and we embed a diffuse gaseous halo inside the dark halo of the primary. Such a halo is expected as a by-product of the process of galaxy formation and has a density and temperature consistent with observational constraints (see Supplementary Information).

With an orbital time of about 1.7 Gyr the dwarf undergoes as many as 5 pericenter passages in 10 Gyr. At the first pericenter passage its dark halo loses $60 \%$ of its mass. The disk, deep inside the potential well of the halo, suffers no stripping, but the tidal perturbation triggers a strong bar instability (Figure 1) and simultaneously heats the stars in the disk. The bar funnels most of the gas towards the central kiloparsec. Gas will be removed from the galaxy if the ram pressure force exerted by the diffuse hot gas, which is proportional to $\rho_{\mathrm{g}} V^{2}$ ( $\rho_{\mathrm{g}}$ being the density of the gaseous halo and $V$ being the orbital speed of the galaxy), exceeds the gravitational restoring force provided by the potential well of the galaxy ${ }^{23}$. Ram pressure readily removes the gas outside the bar radius on a timescale of less than $10^{8}$ years, but not the more tightly bound gas inside the bar. When the satellite crosses the pericenter a second time, the new tidal shock lowers the halo density by a factor of 2 inside 1 kiloparsec, so that $V_{\text {peak }}$ drops to less than $30 \mathrm{~km} / \mathrm{s}$. Since the potential well has become shallower, even the gas sitting inside the bar can be swept away by ram pressure (Figure 1, see also Supplementary Information). The cosmic UV background heats and ionizes the gas, which enhances stripping significantly since the higher gas pressure opposes the gravitational restoring force. Once the first two orbits have been completed no gas is retained by the dwarf.

The response of the system to the tides becomes progressively more impulsive at each new pericenter passage. As a result, the initially disky stellar distribution is heated into a nearly spherical, isotropic configuration (Figure 1). The expansion reflects the attempt of the system to gain a new equilibrium as the internal binding energy is lowered. The removal of gas due to ram pressure is crucial for tidal heating to be effective (see Supplementary Information).

After 10 billion years a diffuse spheroidal galaxy has replaced the gas-rich disk. During the last few orbits the stellar velocity dispersion profile is fairly flat (left panel of Figure 2), matching the profiles observed for Draco and Ursa Minor ${ }^{24}$. The initial angular momentum of the stars has been transported outward during the morphological transformation ${ }^{8}$, producing a ratio between rotational velocity and random motions $v_{\text {rot }} / \sigma<0.2$ in the inner few hundred parsecs. The surface brightness and total luminosity resemble those of the faintest dSphs (see caption of Figure 2). A substantial halo is preserved within a few kiloparsecs from the center so that the final $M / L$ is larger than 100 (right panel of Figure 2 ). The central dark matter density, which has decreased by almost a factor of 4 since the beginning of the simulation, is $\sim 0.2 M_{\odot} / \mathrm{pc}^{3}$, comparable to that of Draco and Ursa Minor $^{1}$.

We predict that all massive galaxies should have a few extremely dark matter dominated satellites as the mechanism reported here is completely general within hierarchical structure 

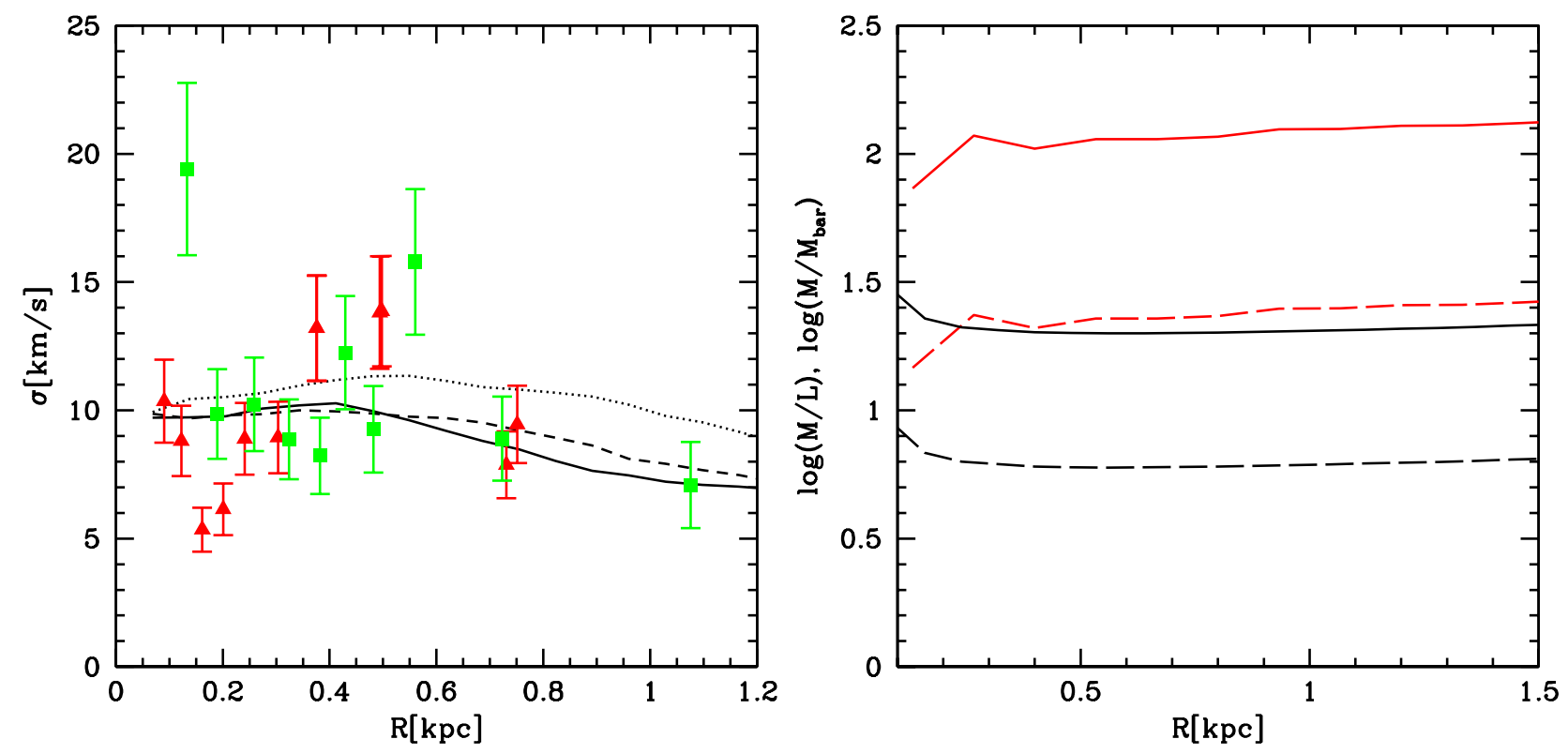

Figure 2. Structural properties of the simulated dwarf after 10 billion years of evolution. Left: The line-of-sight stellar velocity dispersion profiles are shown for three random directions (black lines) perpendicular to each other, together with published observational data points for Draco (red triangles) and Ursa Minor (green squares), including formal 1- $\sigma$ error bars 24. The curves are shown out to the radius for which data points are available. Right: Mass profiles shown out to the radius (from the center of the simulated dwarf) at which stars are gravitationally bound. The dashed lines show the initial (black dashed line) and final (red dashed line) ratios of the total mass to the baryonic mass. The solid lines show the initial (black solid line) and final (red solid line) B band mass-to-light ratios of the dwarf. We have assumed stellar mass-to-light ratio $\left(M / L_{B}\right)_{*}=1.5$ (initial) and $\left(M / L_{B}\right)_{*}=5$ (final) to compute the initial and final luminosities, $M_{B}=-12.5$ and $M_{B}=-9$. The final central surface brightness is $\mu_{B} \approx 26$ mag $\operatorname{arcsec}^{-2}$. We note that an initial $\left(M / L_{B}\right)_{*}=1.5$ is motivated by the fact that at $z>2$ a stellar population is at most three billion years old, whereas a final $\left(M / L_{B}\right)_{*}=5$ is consistent with passive fading of the stellar population for about ten billion years ${ }^{10}$.

formation. The efficiency of star formation in isolated low-mass galaxies can be lower than that assumed here ${ }^{21}$ (see Supplementary Information), and hence dwarf satellites having gas fractions higher than $80 \%$ of the baryons before stripping probably did exist. Once they lose their gas, these systems will turn into dSphs even more dark matter dominated than Draco and Ursa Minor. They should orbit the Milky Way and M31 and have escaped detection so far owing to their low surface brightness, especially at low Galactic latitude ${ }^{25}$. Their halo masses should be comparable to those of known dSphs and thus might help to solve the missing satellites problem ${ }^{26}$. The Ursa Major dwarf recently detected by the Sloan Digital Sky survey ${ }^{27,28}$, which has a halo as massive as that of Draco but a luminosity a hundred times smaller, is probably one of them. Three more systems such as Ursa Major would be enough to bring theory and observations in agreement at the high end of the mass 
function of satellites, corresponding to a peak circular velocity of around $25-30 \mathrm{~km} / \mathrm{s}$, where suppression of baryonic infall by reionization is hardly effective.

When the ultraviolet background is an order of magnitude weaker, as is predicted ${ }^{22}$ at $z<1$, about $30 \%$ of the original gas component stays in the galaxy (see Supplementary Information). Therefore dwarfs that fell into the Milky Way halo late can continue forming stars, and tidal shocks will produce periodic bursts of star formation ${ }^{10}$. These newcomers should account for those dSphs that have fairly normal mass-to-light ratios, extended star formation histories and larger distances from the primaries ${ }^{1,2}$. This explains why Fornax is ten times brighter than Draco and has a very different star formation history despite having a comparable depth of the potential well ${ }^{1,6,29}$. It implies that there should be a positive correlation between $M / L$ and the infall epoch of dwarfs, and thus a negative correlation between $M / L$ and their orbital time. The dSph Tucana represents the biggest challenge to our model because it lies far from any massive galaxy (see Supplementary Information). The accurate determination of the orbits of the dwarfs expected from on going and future astrometric missions such as the Space Inteferometry Mission and the Global Astrometric Interferometer for Astrophysics will be able to test this prediction.

Received; Accepted .

1. Mateo, M. Dwarf Galaxies of the Local Group. Annual Review of Astronomy and Astrophysics. 36, 435-506 (1998)

2. Grebel, E. K. in The Stellar Content of Local Group Galaxies (eds Whitelock, P. \& Cannon, R.) 17-38, (IAU Symposium 192, ASP, San Francisco 1999)

3. Kleyna, J.T., Wilkinson, M.I., Evans, N.W., Gilmore, G. \& Frayn, C. Dark matter in dwarf spheroidals - II. Observations and modeling of Draco. Mon. Not. R. Astron. Soc. 330, 778-791, (2002)

4. Chapman, S., Ibata, R., Lewis, G., Fergusom, A., Irwin, M., McConnachie, A. \& Tanvir, N. A Keck DEIMOS Kinematic Study of Andromeda IX: Dark Matter on the Smallest Galactic Scales. Astrophys. J. 632, L87-L90 (2005)

5. Gallagher, J. S., Madsen, G. J., Reynolds, R. J., Grebel, E. K. \& Smecker-Hane, T. A. A Search for Ionized Gas in the Draco and Ursa Minor Dwarf Spheroidal Galaxies. Astrophys. J. 588, 326-330 (2003).

6. Kazantzidis, S., Mayer, L., Mastropietro, C., Diemand, J., Stadel, J. \& Moore, B. Density profiles of Cold Dark Matter substructure: implications for the missing-satellites problem. Astrophys. J. 608, 663-679 (2004)

7. Lin, D.N.C. \& Faber, S.M. Some implications of nonluminous matter in dwarf spheroidal galaxies. Astrophys. J. 266, L21-L25 (1983)

8. Dekel, A. \& Silk, J. The origin of dwarf galaxies, cold dark matter, and biased galaxy for mation. Astrophys. J. 303, 39-55 (1986) 
9. Bullock, J., Kravtsov, A. \& Weinberg, D.H. Reionization and the abundance of galactic satellites. Astrophys. J. 539, 517-521 (2000)

10. Mayer, L., Governato, F., Colpi, M., Moore, B., Quinn, T., Wadsley, J., Stadel, J. \& Lake, G. The Metamorphosis of Tidally Stirred Dwarf Galaxies. Astrophys. J. 559, 754-784 (2001)

11. de Blok, W.J.G. \& McGaugh, S.S. The dark and visible matter content of low surface brightness disc galaxies. Mon. Not. R. Astron. Soc. 290, 533-552 (1997)

12. Mayer, L. \& Moore, B, The baryonic mass-velocity relation: clues to feedback processes during structure formation and the cosmic baryon inventory. Mon. Not. R. Astron. Soc. 354, 477-484 (2004)

13. Susa, H. \& Umemura, M. Formation of Dwarf Galaxies during the Cosmic Reionization. Astrophys. J. 600, 1-16 (2004)

14. Mac Low, M.M. \& Ferrara, A. Starburst-driven Mass Loss from Dwarf Galaxies: Efficiency and Metal Ejection. Astrophys. J. 513, 142-155 (1999)

15. Einasto, J., Kaasik, A. \& Saar, E. Dynamic evidence on massive coronas of galaxies. Nature. 252, 111-113 (1974)

16. Marcolini, A., Brighenti, F. \& D'Ercole, A. Three-dimensional simulations of the interstellar medium in dwarf galaxies - I. Ram pressure stripping. Mon. Not. R. Astron. Soc. 345, 1329$1339(2003)$

17. Mori, M. \& Burkert, A. Gas stripping of dwarf galaxies in clusters of galaxies, Astrophys. J. 538, 559-568 (2000)

18. Kravtsov, A. V., Gnedin, O. Y.\& Klypin, A. A. The Tumultuous Lives of Galactic Dwarfs and the Missing Satellites Problem. Astrophys. J. 609, 482-497 (2004)

19. Diemand, J., Madau, P. \& Moore. B. The distribution and kinematics of early high-sigma peaks in present-day haloes: implications for rare objects and old stellar populations. Mon. Not. R. Astron. Soc. 364, 367-383 (2005)

20. Governato, F., Mayer, L., Wadsley, J., Gardner, J. P., Willman, Beth, Hayashi, E., Quinn, T., Stadel, J.\& Lake, G. The Formation of a Realistic Disk Galaxy in Lambda-dominated Cosmologies. Astrophys. J. 607, 688-696, 2004

21. Verde, L., Oh, P.S. \& Jimenez, R. The abundance of dark galaxies.Mon. Not. R. Astron. Soc. 336, 541-549 (2002)

22. Haardt, F. \& Madau, P. Radiative Transfer in a Clumpy Universe. II. The Ultraviolet Extragalactic Background. Astrophys. J. 461, 20-37 (1996)

23. Gunn, J. E. \& Gott, J. R. I. On the Infall of Matter Into Clusters of Galaxies and Some Effects on Their Evolution. Astrophys. J. 176, 1-19 (1972)

24. Munoz, R.P. et al. Exploring Halo Substructure with Giant Stars: The Velocity Dispersion Profiles of the Ursa Minor and Draco Dwarf Spheroidal Galaxies at Large Angular Separations. Astrophys. J. 631, L137-L141 (2005) 
25. Willman, B., Governato, F. Dalcanton, J.J., Reed, D. \& Quinn, T. The observed and predicted spatial distribution of Milky Way satellites. Mon. Not. R. Astron. Soc. 353, 639-646 (2004)

26. Moore, B., Ghigna, S., Governato, F., Lake, G., Quinn, T., Stadel, J. \& Tozzi, P. Dark Matter Substructure within Galactic Halos. Astrophys. J. 524, L19-L22 (1999)

27. Willman, B. et al. A New Milky Way Dwarf Galaxy in Ursa Major. Astrophys. J. 626, L85-L88 (2005)

28. Kleyna, J.T., Wilkinson, M.I., Evans, Wyn N., \& Gilmore, G. Ursa Major:a missing low mass CDM halo?. Astrophys. J. 630, L141-L144 (2005)

29. Walker, M .G., Mateo, M., Olszewski, E.W., Bernstein, R., Wang, X. \& Wooodrofe, M., Internal Kinematics of the Fornax Dwarf Spheroidal Galaxy, Astron. J. 131, 2114-2139 (2006)

\section{Acknowledgements}

Stimulating discussions with Y. Birinboim, J. Bullock, A. Dekel, F. Governato, A. Kravtsov, G. Lake, C. Porciani, J. Penarrubia, M. Valluri, B. Willman, and A. Zentner are greatly acknowledged. We thank Steve Majewski and Ricardo Munoz for sharing their data with us. This research has been supported by the Zwicky Prize Fellowship program at the Swiss Federal Institute of Technology in Zurich and by the U.S. Department of Energy through a KIPAC Fellowship at Stanford University and the Stanford Linear Accelerator Center. This project was also supported in its initial stages by the Swiss National Science Foundation and the Kavli Institute for Cosmological Physics (KICP) at the University of Chicago. L. Mayer acknowledges KICP for hospitality during the initial stages of this project. L. Mayer and S. Kazantzidis are grateful to the Aspen Center for Physics for an invitation to the summer workshop "Deconstructing the Local Group - Dissecting Galaxy Formation in our Own Background" where some of this work was completed. All computations were performed on the Zbox supercomputer at the University of Zürich, on LeMieux at the Pittsburgh Supercomputing Center, and on the Gonzales cluster at ETH Zürich.

Author Information The authors declare that they have no competing financial interests. Correspondence and requests for materials should be addressed to L.M. (lucio@phys.ethz.ch) or S.K. (stelios@slac.stanford.edu). 
The origin of the darkest galaxies in the Universe

\section{SUPPLEMENTARY INFORMATION}

Here we briefly describe the setup of the initial conditions and the numerical methods used to perform the simulations presented in the Letter. This is followed by a discussion of the tests and the analysis performed in order to verify and understand the results in depth.

\section{Simulation code}

We have used the fully parallel, N-body+smoothed particle hydrodynamics (SPH) code GASOLINE to compute the evolution of both the collisionless and dissipative component in the simulations. A detailed description of the code is available in the literature ${ }^{1}$. Here we recall its essential features. GASOLINE computes gravitational forces using a tree$\operatorname{code}^{2}$ that employs multipole expansions to approximate the gravitational acceleration on each particle. A tree is built with each node storing its multipole moments. Each node is recursively divided into smaller subvolumes until the final leaf nodes are reached. Starting from the root node and moving level by level toward the leaves of the tree, we obtain a progressively more detailed representation of the underlying mass distribution. In calculating the force on a particle, we can tolerate a cruder representation of the more distant particles leading to an $O(N \log N)$ method. Since we only need a crude representation for distant mass, the concept of "computational locality" translates directly to spatial locality and leads to a natural domain decomposition. Time integration is carried out using the leapfrog method, which is a second-order symplectic integrator requiring only one costly force evaluation per timestep and only one copy of the physical state of the system. In cosmological simulations periodic boundary conditions are mandatory. GASOLINE uses a generalized Ewald method ${ }^{3}$ to arbitrary order, implemented through hexadecapole.

$\mathrm{SPH}$ is a technique of using particles to integrate fluid elements representing gas $^{4,5}$. GASOLINE is fully Lagrangian, spatially and temporally adaptive and efficient for large $N$. The version of the code used in this Letter includes radiative cooling and accounts for the effect of a uniform background radiation field on the ionization and excitation state of the gas. The cosmic ultraviolet background is implemented using the HaardtMadau model ${ }^{7}$ which includes photoionizing and photoheating rates produced by QSOs and galaxies starting at $z=7$. The assumption that reionization occurred at $z=7$ is conservative but consistent with the combination of the 3rd year WMAP results and the Gunn-Peterson effect in the spectra of distant quasars ${ }^{8}$. We use a standard cooling function for a primordial mixture of atomic hydrogen and helium (the metallicity in dSphs is indeed much lower than solar ${ }^{9}$, with $-1<[\mathrm{Fe} / \mathrm{H}]<-2$ ). The cooling shuts off below $10^{4} \mathrm{~K}$. The internal energy of the gas is integrated using the asymmetric formulation, that gives results very close to the entropy conserving formulation ${ }^{6}$ but conserves energy better. Dissipation in shocks is modeled using the quadratic term of the standard Monaghan artificial viscosity ${ }^{5}$. The Balsara correction term is used to reduce unwanted shear viscosity ${ }^{10}$. 


\section{Initial Conditions: the cosmological simulation}

The cosmological simulation employs the so called volume renormalization technique to achieve higher resolution in a region of interest (described in the next section). It is one of the simulations already presented elsewhere ${ }^{11,16}$. The virial mass of the halo that was selected at $z=0$ to be re-simulated at higher resolution is $1.15 \times 10^{12} M_{\odot}$ (the virial mass is measured within the virial radius $R_{\text {vir }}$, the radius enclosing an overdensity of 100 times the critical density $\left.\rho_{\text {crit }}\right)$. A recent analysis of all observational constraints points to a Milky Way halo of about $10^{12} M_{\odot}{ }^{11}$. The halo was originally selected within a low resolution, dark matter only simulation run in a concordance, flat, $\Lambda$-dominated cosmology: $\Omega_{0}=0.3$, $\Lambda=0.7, h=0.7, \sigma_{8}=0.9$, shape parameter $\Gamma=0.21$, and $\Omega_{b}=0.039^{12}$. The size of the box, $100 \mathrm{Mpc}$, is large enough to provide realistic torques. The power spectra to model the initial linear density field were calculated using the CMBFAST code to generate transfer functions. Dark matter particle masses in the high resolution regions are $8.05 \times 10^{5} M_{\odot}$, and and the force resolution, i.e. the gravitational softening, is $0.3 \mathrm{kpc}$. In total there are $1.4 \times 10^{6}$ particles within the virial radius.

With our choices of particle number and softening, the smallest subhalos resolved have typical circular velocities of $10 \%$ of their host. For all particle species in the high resolution region, the gravitational spline softening, $\epsilon(z)$, evolved in a comoving manner from the starting redshift $(\mathrm{z} \sim 100)$ until $\mathrm{z}=9$, and remained fixed at its final value from $\mathrm{z}=9$ to the present. The softening values chosen are a good compromise between reducing two body relaxation and ensuring that disk scale lengths and the central part of dark matter halos will be spatially resolved. Integration parameter values were chosen according to the results of previous systematic parameter studies ${ }^{14}$.

The merging histories and angular momentum of the parent dark matter halo are supposed to play a major role in defining the final properties of the galaxies that form inside them. It is then important to make sure that our halos have merging histories and spin parameters somewhat representative of the global population. The halo was selected with the only criteria that the redshift of the last major merger $\left(\mathrm{z}_{l m m}\right)$ was $>2$ and that there are no halos of similar or larger mass within a few virial radii (a major merger is defined here as having a 3:1 mass ratio). The halo spin parameter, $\lambda$, is $\sim 0.05$ at $z=0$, close to the average value for cosmic halos $\sim 0.035^{15}$. Cosmological simulations with hydrodynamics that use this same and other similar initial conditions show that realistic disk galaxies similar to the Milky Way do arise in these dark matter halos ${ }^{16}$.

\subsection{Volume renormalization technique}

The large dynamic range involved in cosmological simulations aimed at resolving the scale of galactic halos calls for techniques that concentrate the computational power on the

object of interest. This is achieved by the volume renormalization technique ${ }^{17}$. A large scale simulation is done at low resolution and the regions of interest are identified, e.g. 
QSOs forming at high redshift, a large cluster of galaxies or a Milky Way-sized halo such as in this Letter. Next, initial conditions are reconstructed using the same low-frequency waves present in the low resolution simulation but adding the higher spatial frequencies. To reduce the number of particles and make the nonlinear simulation possible with the same cosmological context, we construct another set of initial conditions with particles whose mass, and therefore mean separation, increase with the distance from the center of our volume. Note that because tides are important in the formation of the filaments it is not sufficient to extract just the central region. Generating initial conditions (ICs) in this approach is nontrivial. The ICs are calculated on a grid determined by the initial mass resolution. The power spectrum is realized by using Fast Fourier Transforms (FFTs) to determine displacements of particles from this grid. We use a particular technique that allows one to calculate high resolution FFTs only in the regions of the simulations were mass and force resolution need to be high. This method has been successfully used in a wide range of cosmological studies ${ }^{18,19,20,11}$.

\section{Initial Conditions: the dwarf galaxy model}

The multi-component dwarf galaxy models are built using a standard technique that produces initial conditions very close to equilibrium ${ }^{20,21}$. The model is further evolved in isolation for several Gyr in order to remove possible transients caused by initial particle noise, and the end state is used as initial condition. The scheme we use to assign the structural parameters of the baryons in a dark matter halo assumes that baryons collapse into the halo having the same specific angular momentum distribution of the halo and conserving angular momentum during collapse ${ }^{23}$. The properties of the baryonic component are related to those of the dark halo, namely its mass, angular momentum (via the spin parameter) and concentration. We refer to previous papers for a detailed description of the scaling relations that relate the baryonic component and the dark halo ${ }^{24,25}$. The technique has been widely used to build models of dwarf galaxies ${ }^{26,27}$.

The bayonic component comprises an exponential disk of gas and stars embedded in an NFW halo ${ }^{28}$. The halo has $V_{\text {peak }}=40 \mathrm{~km} / \mathrm{s}$. In CDM models low mass halos have on average higher concentration parameters $c=R_{\text {vir }} / r_{\mathrm{s}}$ (where $R_{\mathrm{vir}}$ is the virial radius and $r_{\mathrm{s}}$ the scale radius) relative to higher mass halos and, for a given halo mass, the concentration decreases with redshift roughly as $(1+z)^{-1}$. We choose $c=20$ for the halo of the dwarf. This value of the halo concentration matches the average concentration of the subhalos with $V_{\text {peak }}$ in the range $30-40 \mathrm{~km} / \mathrm{s}$ in the cosmological simulation before they fall into the primary halo and is consistent with the typical values expected at these mass scales at $z \sim 2$, as shown elsewhere ${ }^{29}$. In choosing the radius and mass of the disk there is some freedom. However, there are constraints coming from the link between disk radius and the spin parameter of the halo in which the disk forms, and from the information of the typical fraction of the mass of present-day dwarf galaxies that is contributed by the baryonic disk. The first constraint brackets the range of possible disk radii at given disk 

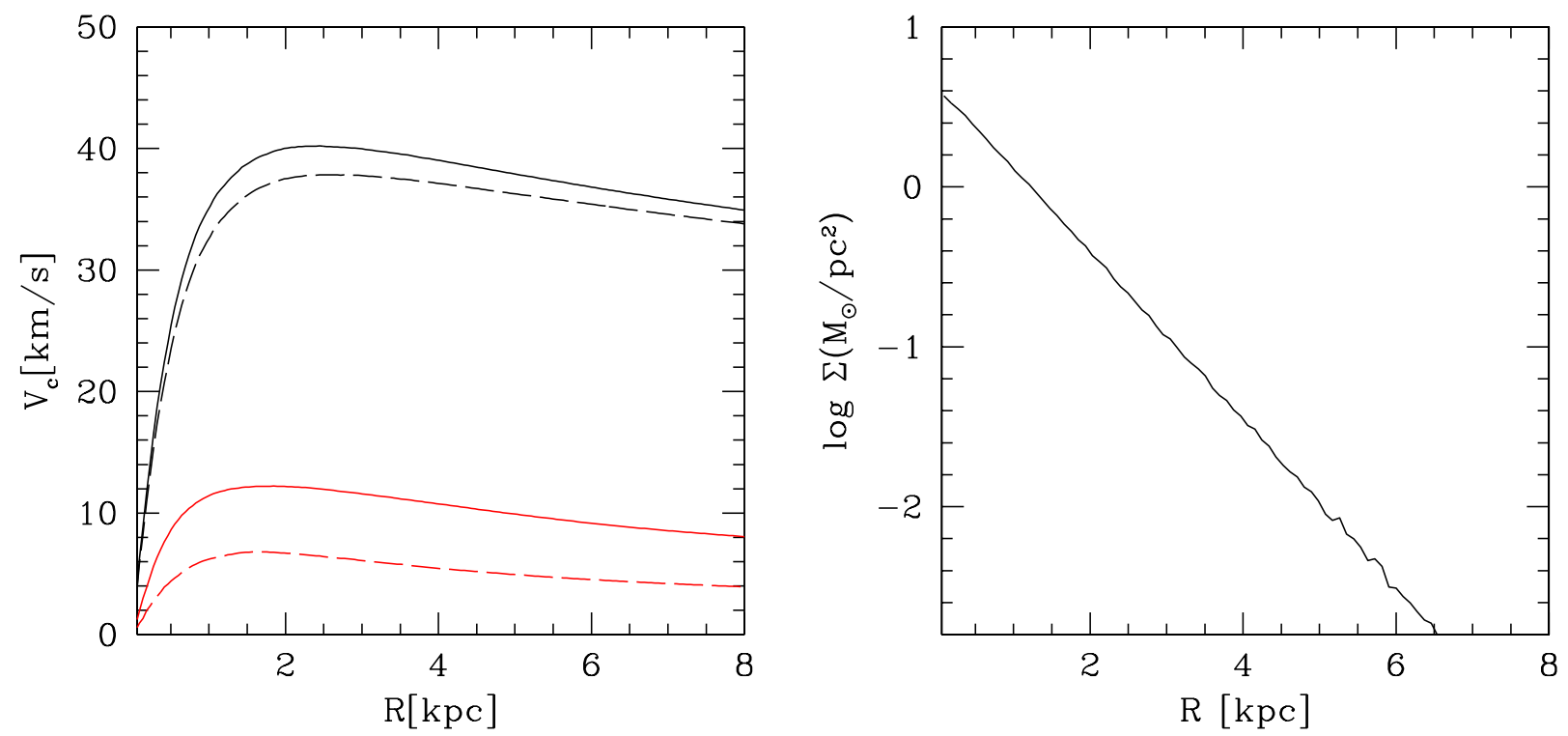

Figure 3. Structural properties of the initial dwarf galaxy model. The left panel shows the initial rotation curve. The red solid line shows the contribution of the gas, the red dashed line the contribution of the stars, the black dashed line shows the dark matter contribution and the black solid line shows the total rotation curve resulting from the sum of the contribution of the different components. The right panel shows the initial baryonic surface density profile.

mass, the second the range of possible disk masses for a given halo mass. Since our simple working hypothesis is that the progenitors of dwarf spheroidals had baryon fractions in the range of the normal values of galaxies in the present-day Universe, we fix the disk mass fraction to $f_{d}=0.035 M_{\text {vir }}$. This value is typical in mass models that reproduce dwarf and low surface brightness galaxies ${ }^{29}$ and is consistent with the baryonic Tully-Fisher relation for galaxies with rotational speeds in the range $40-60 \mathrm{~km} / \mathrm{s}^{30}$. The exponential disk scale length is then fixed by the adopted spin parameter, $\lambda=0.05$. The log-normal distribution of spin parameters of cosmological halos peaks at around $0.035^{15}$ but (1) $40 \%$ of the halos have larger spins and (2) modeling of their rotation curves suggests that dwarf galaxies have an average spin larger than the mean value of the galaxy population as a whole ${ }^{29}$. The resulting exponential disk scale length, which is a function of $\lambda$, the halo concentration $c$ and $f_{\mathrm{d}}{ }^{24}$, is $0.55 \mathrm{kpc}$.

The chosen disk mass and radius yield an initial central baryonic surface density $\sim$ $4 M_{\odot} / \mathrm{pc}^{2}$. We choose the stellar mass fraction in such a way that it is consistent with the amount of star formation expected in a galaxy with the assigned structural parameters. Within the standard structure formation paradigm galaxies in halos of $10^{10} M_{\odot}$ or less, namely in the range of our model, should have little or no star formation because their disks are gravitationally stable nearly everywhere ${ }^{31}$, having Toomre parameters $Q>1$. The stability arises because lower mass halos should harbor lighter disks. The latter statement is true if the disk mass fraction either varies little or tends to decrease for decreasing 
halo masses, in agreement with the analysis of galaxy rotation curves across a range of masses $^{30,31}$. Observations show examples of galaxies that are Toomre-stable and yet have on-going or recent star formation, albeit at a fairly low level ${ }^{32,33}$. Recent models can account even for such departures from the Toomre criterion $^{34,35}$. In one such model the threshold for star formation is determined by the minimum surface density required for molecular gas to form ${ }^{34}$. In our dwarf galaxy model about $1.5 \times 10^{7} M_{\odot}$ of baryons have surface densities above the predicted critical surface density predicted, which is $\sim 2 M_{\odot} / \mathrm{pc}^{2}$. We thus assign to our model a stellar mass of $1.5 \times 10^{7} M_{\odot}$, corresponding to only $20 \%$ of the baryons. Since the critical surface density for star formation can rise by up to a factor of 5 in presence of ionizing radiation fields such as the cosmic ultraviolet background ${ }^{34}$, the assumed stellar mass fraction should be viewed as an upper limit for a reasonable progenitor at these mass scales. Although the criterion based on surface density is convenient, it is known to have difficulties in reproducing some observations. Therefore we compared our choice with another, more recent work that predicts the stellar fraction using fully self-consistent Nbody + SPH simulations with a physically motivated star formation recipe and sink particles to represent sites where molecular clouds and star clusters would form ${ }^{35}$. These simulations employ models of galactic disks with a variety of initial structural parameters, gas mass fractions and gas temperatures. They explore the dependence of the star formation rate on the initial minimum Toomre parameter of the combined gaseous and stellar disk, $Q_{s g}$, and find that star formation is possible up to values of $Q_{s g} \sim 1.6$, significantly larger than the standard threshold $Q_{g} \sim 1$, where $Q_{g}$ is the local Toomre parameter of the gas. Moreover, by running a large set of simulations for about 2 Gyr, they derive a relation between the minimum initial Toomre parameters of the systems, $Q_{s g}(\mathrm{~min})$ or $Q_{g}(\mathrm{~min})$, and the total amount of gas that can be converted into stars. We compared our initial setup with their results. Assuming a fully gaseous disk at the start, our initial galaxy model would have $Q_{s g}(\min )=Q_{g}(\min ) \sim 1.42$ (reached between 1 and 2 disk scale lengths). For such a value of $Q_{g}(\mathrm{~min})$, Figure 15 of their paper (bottom panel) indicates that between $10 \%$ and $17 \%$ of the initial gas should be converted into stars $(13 \%$ if we compare with their best fitting curve). These fractions are close to the $20 \%$ that we assumed, though somewhat smaller; indeed, as we emphasize in the Letter, a smaller initial stellar fraction would make our scenario even stronger. The rotation curve and initial baryonic surface density profile of the dwarf galaxy model are shown in Figure 3.

We choose the mass and force resolution in the dwarf galaxy models in such a way that possible numerical artifacts are under control. We adopt $2 \times 10^{5}$ particles in the gaseous disk and $10^{6}$ particles in the stellar disk and dark matter halo, for a total of $2.2 \times 10^{6}$ particles. The spatial resolution of the dark halo and stars is set by the gravitational softening which is equal to $35 \mathrm{pc}$. The spatial resolution in the gas component is given by the SPH smoothing length, defined as the radius of the spherical kernel adopted by GASOLINE, which contains 32 particles. The smoothing length is adaptive in space and time. In the initial conditions it is about $25 \mathrm{pc}$ at about one disk scale length, and it drops to $\sim 15 \mathrm{pc}$ during the stage of the bar driven gas inflow close to the first pericenter passage. Since most of the mass in an exponential disk is contained within about 3 scale lengths, or $1.5 \mathrm{kpc}$, the gaseous disk 
is resolved by about 60 resolution elements initially; later, as the bar forms, the average smoothing length decreases, but also the size of the gas component becomes smaller by almost a factor of 2 (due to the gas inflow and stripping of the outer regions), so that effectively the resolution remains close to 60 smoothing kernels. This resolution is above that required to properly model ram pressure stripping based on previous works which found that 50 resolution elements in a linear dimension (or 25 along a radius) is needed ${ }^{36,37}$. Moreover, both the smoothing length and the gravitational softening of the gas are at all times much smaller than the local Jeans length, and have comparable values. This ensures that gravity and pressure forces are correctly balanced at the Jeans length, thus avoiding spurious fragmentation or artificial suppression of collapse ${ }^{38}$. The Jeans length is defined

as $\lambda_{J}=\sqrt{\frac{\pi v_{s}^{2}}{G \rho_{0}}}$, where $v_{s}$ is the sound speed, $G$ is the gravitational constant and $\rho_{0}$ is the (local) density. It is smallest during bar formation since the temperature of the bound gas is $\sim 2 \times 10^{4} \mathrm{~K}$ at all times while the density peaks at this time, being close to $10^{-23}$ $\mathrm{g} / \mathrm{cm}^{3}$ near the center. Yet even in this extreme situation $\lambda_{J} \sim 300 \mathrm{pc}$, i.e. about an order of magnitude larger than either twice the SPH smoothing length or the gravitational softening $^{38}$.

\section{Initial Conditions: the Milky Way halo model}

The live dark halo model used for the Milky Way is based on the Milky-Way sized halo of the cosmological simulation (see above). We use a slightly larger value of the circular velocity at the virial radius, $V_{\text {vir }}=180 \mathrm{~km} / \mathrm{s}$, in order to have $V_{\text {peak }}=230 \mathrm{~km} / \mathrm{s}$ at about $10 \mathrm{kpc}$ from the center without including a baryonic disk component. The concentration is $c=10$. With our choice of parameters the shape of the rotation curve is quite flat out to $100 \mathrm{kpc}$ and the resulting halo mass within $100 \mathrm{kpc}$ is $\sim 8 \times 10^{11} M_{\odot}$, consistent with the orbital dynamics of the Magellanic Clouds and distant dSphs ${ }^{39,40}$. We use $10^{6}$ particles to model the halo and a softening of $300 \mathrm{pc}$, as in the cosmological simulation. We embed a gaseous halo in hydrostatic equilibrium within the live dark matter halo. The density of the gaseous halo near the pericenter of the orbit of the satellite, $30 \mathrm{kpc}$, is $2 \times 10^{-4} \mathrm{~cm}^{-3}$, comparable to that of the diffuse gas in recent hydrodynamical cosmological simulations that follow the formation of a disk galaxy inside the same halo used in this paper $^{16}$. Indirect estimates of the present-day density of the gaseous corona surrounding the Milky Way based on OVI,OVII and X-ray absorption measurements are also in the same range $\left(\sim 10^{-4} \mathrm{~cm}^{-3}\right.$ at a distance of $\left.50 \mathrm{kpc}^{42}\right)$. The temperature of the halo is $10^{6}$ $\mathrm{K}$ at $50 \mathrm{kpc}$, which is consistent with the same observational constraints. Assuming an isotropic model, the halo temperature at a given radius $r$ is determined by the cumulative mass distribution $M(r)$ of the dark, stellar and gaseous components of the Milky Way beyond $r$ and by the density profile $\rho_{h}(r)$ of the hot $\operatorname{gas}^{27,43}$

$$
T(r)=\frac{m_{p}}{k_{B}} \frac{1}{\rho_{h}(r)} \int_{r}^{\infty} \rho_{h}(r) \frac{G M(r)}{r^{2}} d r
$$


where $m_{p}$ is the proton mass, $G$ and $k_{B}$ are the gravitational and Boltzmann constants. In reality, the gaseous halo will not be in hydrostatic equilibrium since it would slowly cool and sink to the center while at the same time new gas flows within the virialized region from outside. However, cosmological hydrodynamical simulations ${ }^{16}$ indicate that the characteristic density of the halo at $30-50 \mathrm{kpc}$ from the center does not change significantly from $z=2$ to $z=0$.

\section{Tests of the results}

\subsection{Tidal heating and gas removal}

We have performed a number of tests to explore in detail the nature of the transformation of the baryonic component. The main goal was to determine the main cause of the expansion and roundening of the stellar component. We investigated the relative role of the rapid removal of the gas, which comprises most of the baryons initially, and that of the repeated tidal shocks in changing the shape of the stellar component. Although the initial dwarf model is dark matter dominated by construction, baryons comprise as much as $30 \%$ of the total mass within the initial exponential disk scale length. Their rapid removal due to ram pressure stripping might in principle affect the dark matter potential, possibly lowering its density as found in previous studies where baryonic stripping occurs as a result of powerful supernovae winds $\mathrm{s}^{43,44,45}$.

Figure 4 shows principle axis ratios $s=b / a$ and $q=c / a(a>b>c)$ of stars, calculated from the eigenvalues of a modified inertia tensor ${ }^{46}: I_{i j}=\sum_{\alpha} x_{i}^{\alpha} x_{j}^{\alpha} / r_{\alpha}^{2}$, where $x_{i}^{\alpha}$ is the $i$ coordinate of the $\alpha$ th particle, $r_{\alpha}^{2}=\left(y_{1}^{\alpha}\right)^{2}+\left(y_{2}^{\alpha} / s\right)^{2}+\left(y_{3}^{\alpha} / q\right)^{2}$, and $y_{i}^{\alpha}$ are coordinates with respect to the principle axes. We use an iterative algorithm starting with a spherical configuration $(a=b=c)$ and use the results of the previous iteration to define the principle axes of the next iteration until the results converge to a fractional difference of $10^{-2}$. Results are shown for three experiments. The comparison between the red and the black lines clearly shows that repeated tidal shocks are necessary for the transformation of the stellar component to occur; the increase of $c / a$ is amplified by more than a factor of 6 when the dwarf is continuously tidally shocked relative to a case in which it just loses most of its baryons owing to ram pressure and then evolves without any tidal perturbation (in the latter case we removed the primary halo from the simulation after the gas was stripped). The green lines in Figure 4 show a test in which about $30 \%$ of the initial gas content is retained until the end because the UV background is absent and thus the effect of ram pressure is reduced (we tested that not including the cosmic UV background at all or including it with the low amplitude expected at $z<1$ from the Haardt \& Madau model yields the same result ${ }^{26}$ ). The residual gas is funnelled to the center by the bar, where it forms a very dense knot $^{26}$. We notice that despite the fact that this is a small fraction of the initial gas content it represents a non-negligible contribution to the central potential because it all ends up in the inner $0.5 \mathrm{kpc}$ (corresponding to the initial disk scale length). 


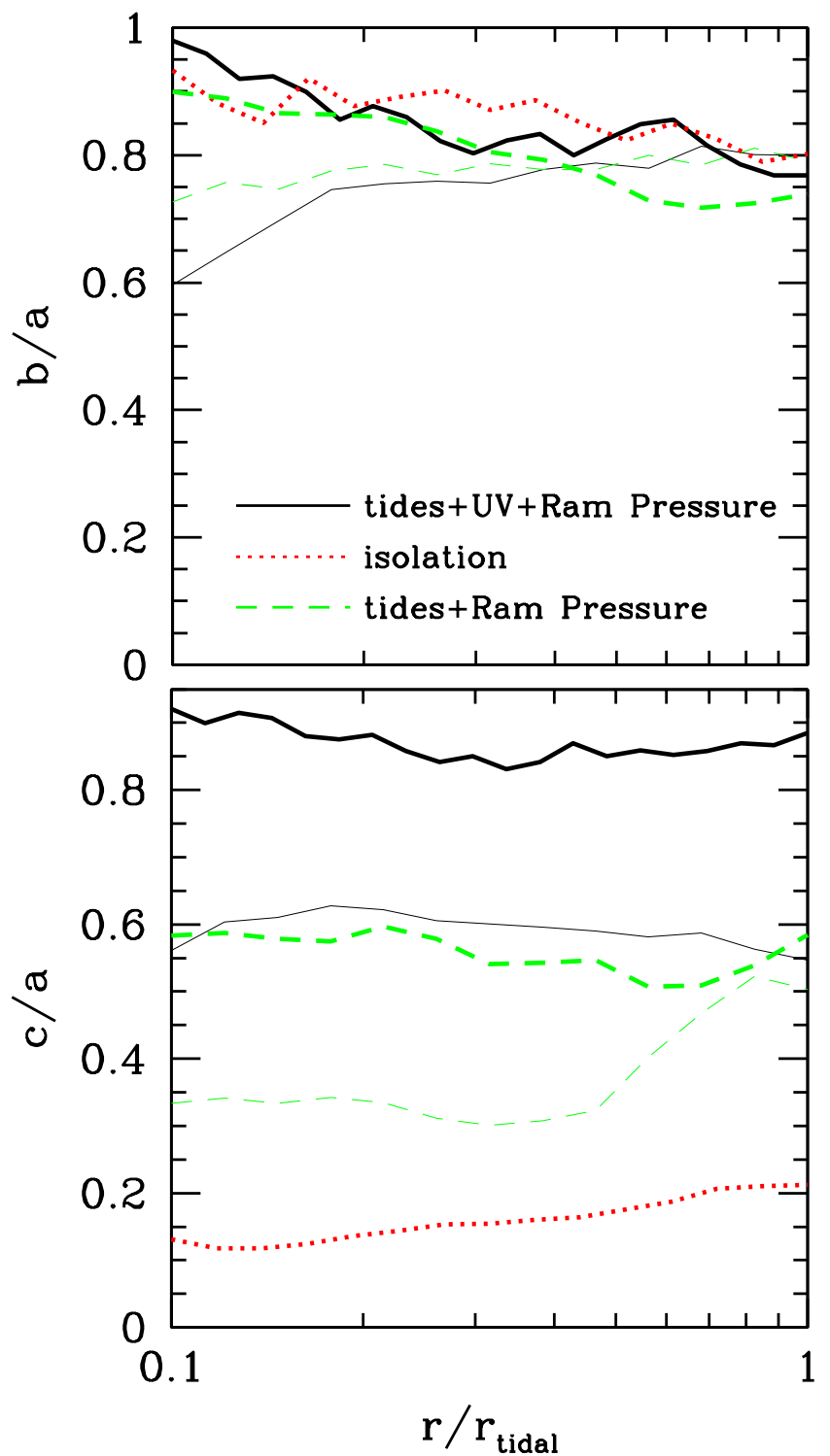

Figure 4. Shape evolution of the stellar component. The upper panel shows the intermediateto-major axis ratio, $b / a$, and the bottom panel presents the minor-to-major axis ratio, $c / a$. Solid lines correspond to the self-consistent simulation in which tides, ram pressure stripping, and UV background were included throughout the orbital evolution of the dwarf. Dotted lines correspond to the case where the dwarf galaxy was extracted from the simulation just after the gas was removed and evolved in isolation. Dashed lines show results of a test in which approximately $30 \%$ of the initial gas mass remains in the galaxy because the absence of the UV background reduces significantly the effectiveness of ram pressure stripping. Thick and thin lines correspond to 10 and 5 Gyr of orbital evolution, respectively (for the extracted dwarf we only show the result after 10 Gyr). The shape of the stellar component changes gradually over time and the presence of UV heating is essential for the shape transformation of the dwarf. 
In this case the dwarf maintains a bar-like, prolate shape even after $10 \mathrm{Gyr}$, hence the lower $c / a$. After 5 Gyr the central density is 8 times higher compared to the simulation in which complete gas stripping occurs. As a result $t_{\text {shock }}>t_{\text {orb }}$ within $0.5 \mathrm{kpc}$, where $t_{\text {shock }}$ is $R_{\text {peri }} / V_{\text {peri }}, R_{\text {peri }}$ and $V_{\text {peri }}$ being, respectively, the pericentric distance and the velocity at pericenter, and $t_{\text {orb }}$ is the orbital time at $0.5 \mathrm{kpc}$ from the center of the dwarf; the response of the system to the tidal forcing is thus adiabatic instead of impulsive, hence further morphological changes are inhibited. This demonstrates that while gas removal does not drive the transformation it represents a crucial step for the evolution of the stellar component because the effect of the tidal shocks is considerably weakened when a dense central gas component is retained.

Figure 4 also shows that $c / a$ does not grow beyond 0.2 in isolation, even after a few Gyr of evolution. The average (apparent) ellipticity measured for late-type dwarfs in the Local Group is larger, $\sim 0.58$, for the 8 dwarfs having a range of rotational velocities comparable with those of our initial dwarf galaxy model $\left(V_{\text {peak }} \sim 30-40 \mathrm{~km} / \mathrm{s}\right)^{47}$. Similar values are found for dwarf irregular galaxies in clusters ${ }^{48}$. However, in comparing with observed values of ellipticity one must take into account inclination effects, since the $c / a$ discussed for our simulated dwarf is the intrinsic value, i.e. the value that would be measured by an observer viewing the dwarf exactly edge-on. By viewing the dwarf at inclinations in the range 30-60 degrees, which statistically are a much more representative situation, the mean apparent $c / a$ varies in the range $\sim 0.4-0.7$ within 3 disk scale lengths (it is 0.55 at 45 degrees of inclination), hence perfectly consistent with the observed values.

The central dark matter density decreases by almost a factor of 4 during the evolution as a result of the repeated tidal shocks when all the gas is readily removed. Since the dwarf is dark matter dominated, it is natural to ask whether the change in the halo potential associated to this drop of the halo central density is sufficient to alter the mass distribution of the stellar component or if the direct heating of the disk by the tidal shocks is crucial. Figure 5 shows thickness of the stellar disk defined as the dispersion of particle positions above the midplane $(z=0)$, as a function of cylindrical radius $R$. The black line corresponds to the initial disk and red line corresponds to the disk thickness after 5 Gyr of evolution in isolation. The good agreement between these two curves suggests that the initial disk model is in reasonable equilibrium. The green line shows instead the results of an experiment with the isolated dwarf galaxy model in which we decreased the mass of the inner dark matter halo over 5 Gyr by the same amount as in the simulation in which the primary halo is included. The decrease of the central dark matter mass is of order of $40 \%$ over 5 Gyr and is responsible for an increase in thickness of less than $30 \%$. The stellar component maintains an evident disk-like structure. Hence the transformation of the disk is not driven by the response to the changing dark matter potential.

In summary, these experiments indicate that the repeated tidal shocks in the impulsive regime are driving the transformation of the shape and mass distribution of the dwarf galaxy and that gas removal is a necessary condition for them to be effective. We note that in previous works on tidal stirring ${ }^{49,50}$ morphological changes were happening primarily as 


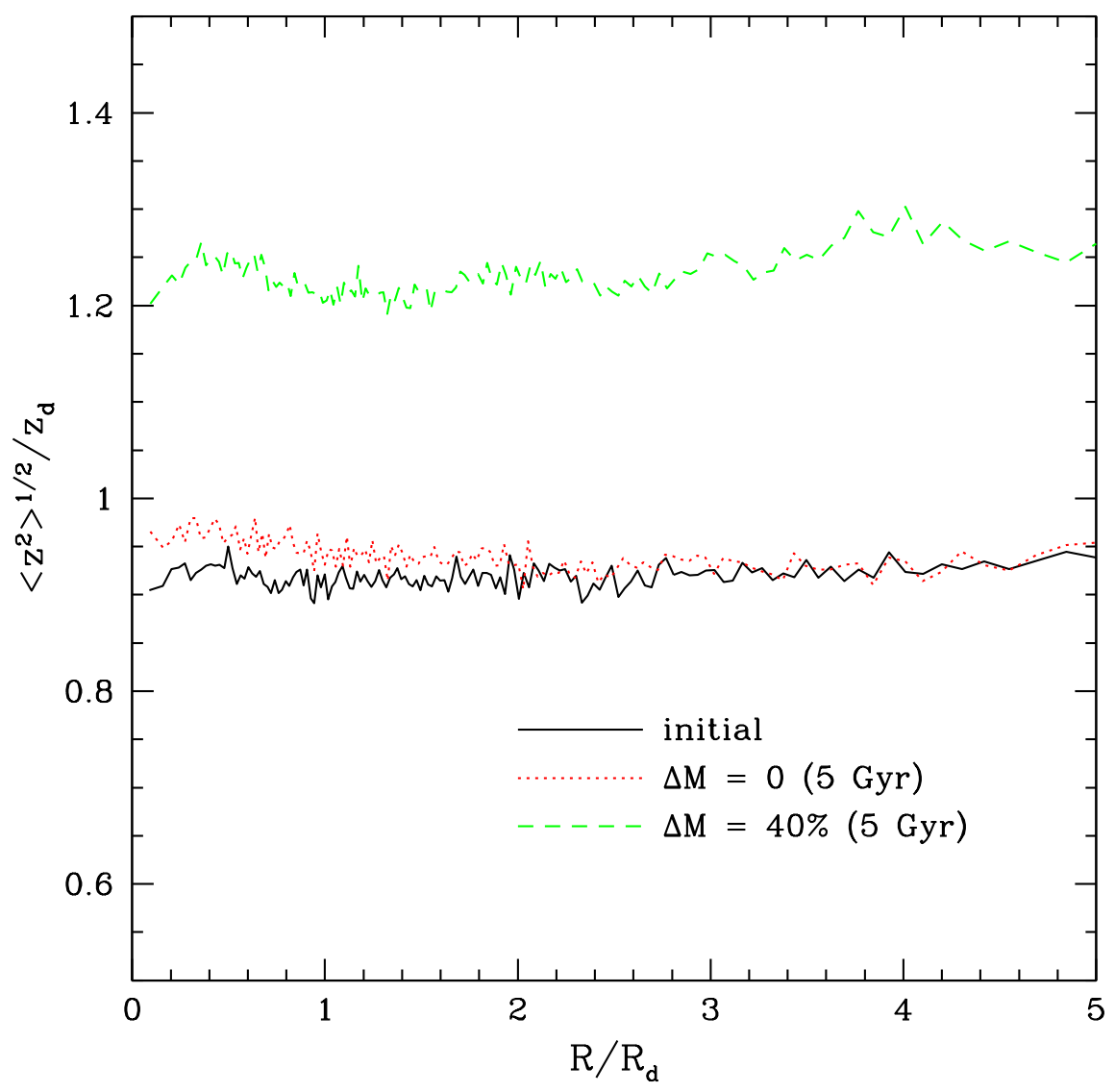

Figure 5. Evolution of the disk thickness as a function of radius, computed relative to the initial disk scale height, $z_{d}$ that characterizes the vertical surface density profile $\left(R_{d}\right.$ is the initial disk scale length). The results are shown for the run in isolation (red line) and for the run in which $40 \%$ of the halo mass within a sphere containing the disk radius is gradually removed over a timescale of 5 Gyr (green line). The initial disk thickness is also shown for comparison (black line).

a result of tidally induced dynamical instabilities rather than impulsive heating. There the buckling of the bar was driving the change of shape from a disk into a spheroid. Here buckling never occurs because the self-gravity of the stars is too low for global bending modes to grow. In fact in those previous works the dwarf models had similar baryonic masses but stellar masses nearly an order of magnitude larger (conversely the gas was at most contributing $30 \%$ of the baryonic mass).

Although tides and non-axisymmetric instabilities heat the system and remove angular momentum ${ }^{49,50}$, some residual rotation is present in the dwarf after ten billion years. Figure 6 shows the intrinsic rotational velocity as a function of radius; this corresponds to the maximum rotational velocity that can be observed, i.e. the velocity measured by observing the dwarf perpendicular to its rotation axis. The rotation is negligible near the center but 


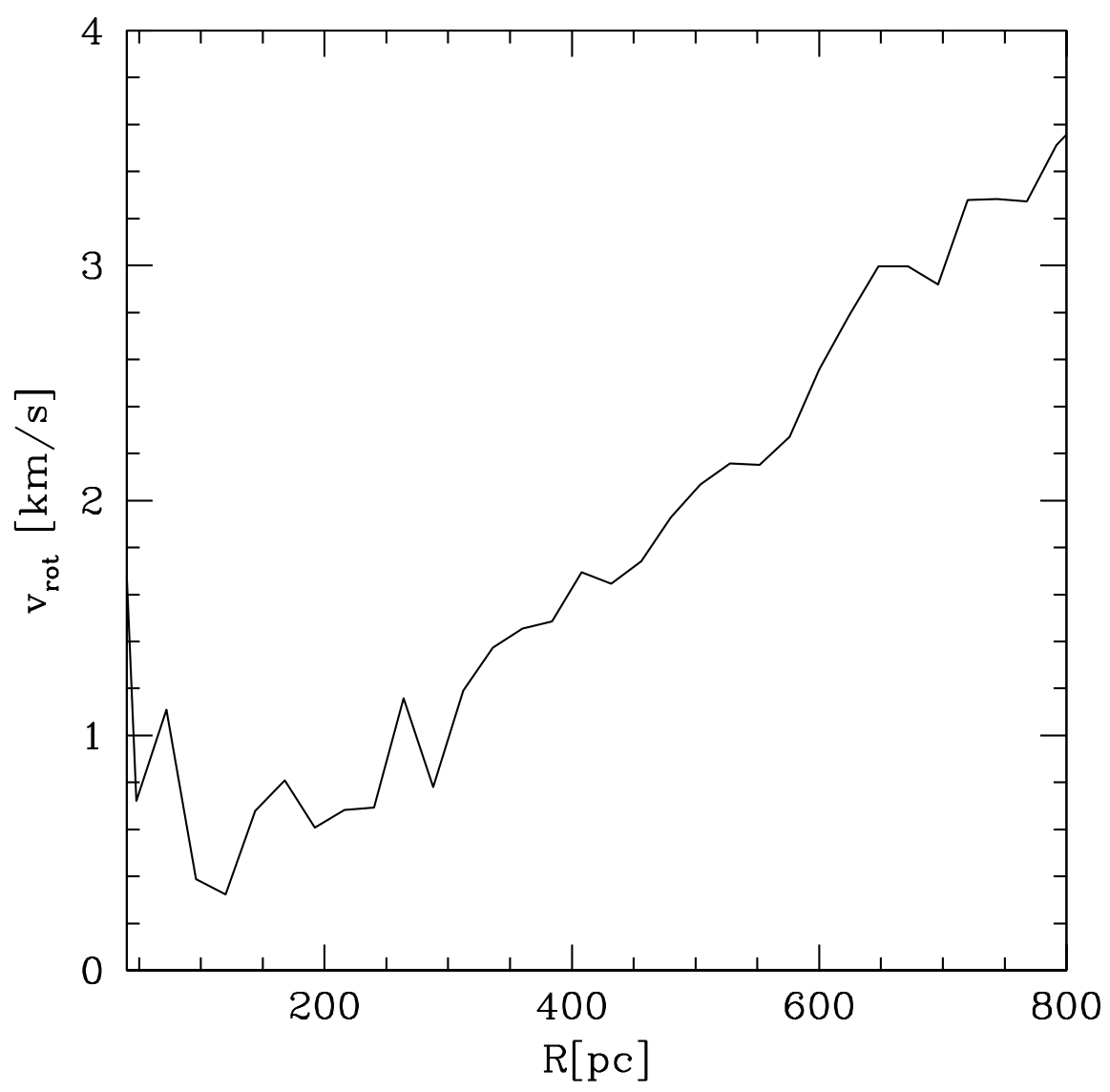

Figure 6. Rotational velocity profile of the stars as a function of the distance from the center of the dwarf after $10 \mathrm{Gyr}$ of evolution. The profile is azimuthally averaged and spherical radial bins have been used.

becomes larger than $\sim 3 \mathrm{~km} / \mathrm{s}$ at several hundred parsecs from the center. Interestingly, this is comparable to the rotation inferred for Ursa Minor ${ }^{51,52}$, for which a velocity gradient around the apparent morphological major axis has been observed out to a distance of a few hundred parsecs. How much such rotation reflects the original rotation of the dwarf and how much it is the result of dynamical evolution driven, for example, by tidal torques, will be subject of a future detailed investigation.

\subsection{Resolution tests on gas density}

Our simulations lack radiative transfer. Self-shielding of the gas could reduce the net heating from the cosmic UV background and therefore reduce the effect of ram pressure. Recently, cosmological simulations with SPH hydrodynamics have been used to study the formation of dwarf galaxies at high redshift, before as well as during the reionization epoch $^{53}$. These include radiative transfer to follow the propagation of UV photons produced by a cosmic ionizing background similar to the one employed here and find that 
the gas self-shields efficiently only for densities $\rho>10^{-22} \mathrm{~g} / \mathrm{cm}^{3}$. The gas has always a density at least an order of magnitude lower than the latter threshold in our simulations; even during the phase in which the density increases the most, namely after the bar-driven gas inflow, we measure $\rho \sim 10^{-23} \mathrm{~g} / \mathrm{cm}^{3}$. We conclude that self-shielding will be negligible given the low density of the gas in the simulated dwarf. However, resolving the highest gas densities correctly is not trivial in numerical simulations. We tested that our inference on the importance of self-shielding is not dependent on the adopted resolution by running the same simulation at three different mass and force resolutions, spanning a factor of 64 in number of particles (the highest resolution run employs 1.6 million gas particles and was run only for one orbit). The results are shown in Figure 7. As it can be seen, between the lowest and the highest resolution case the difference in the maximum gas density is less than a factor of 2 , and it is less than $40 \%$ between the intermediate and highest resolution cases. The intermediate resolution case corresponds to the standard resolution adopted in our simulations. The figure also shows that a decrease of a factor of 3 in the gravitational softening implies an increase in the maximum density of only about $30 \%$ at fixed particle number.

\section{Critical discussion of the results}

\subsection{The various modes of ram pressure stripping}

The gas is first stripped by tides and instantaneous ram pressure as the galaxy approaches pericenter. This removes the outer disk, outside about two initial disk scale lengths. For

such gas, the ram pressure force, $\rho_{g h_{p}} V_{p}^{2}\left(\rho_{g h_{p}}\right.$ is the density of the halo near pericenter and $V_{p}$ is the orbital velocity of the galaxy near pericenter, that are, respectively, $10^{-4}$ atoms $\mathrm{cm}^{-3}$ and $300 \mathrm{~km} / \mathrm{s}$ ) is stronger than the gravitational restoring force, which is mainly provided by the dark halo. More instantaneous ram pressure stripping occurs also at second pericenter as the halo central density, and hence the gravitational restoring force, has decreased in the meantime due to the tidal shocks, and the galaxy loses its gas down to about one initial disk scale length. Quantitative comparisons with analytic predictions ${ }^{54}$ for instantaneous stripping are difficult because tidal forces continuously affect the magnitude of the gravitational restoring force and participate in the stripping, and the gas is subject to compressional heating, radiative cooling and an external radiation field. Nevertheless, it is important to notice, that dwarf galaxy models almost identical to the one simulated here have stripping radii within $30 \%$ of those predicted by the Gunn \& Gott formula ${ }^{54}$ when they are evolved in periodic tube flows that simulate a single pericenter passage in absence of tidal forces ${ }^{26,42}$.

However, the gas inside about one disk scale length lies inside the tidal radius at all times, hence tidal stripping does not play a role; in addition, for such gas the gravitational restoring force is always higher than the instantaneous ram pressure force. Stripping might still occur by ablation via Kelvin-Helmoltz instabilities, the so called turbulent stripping, 


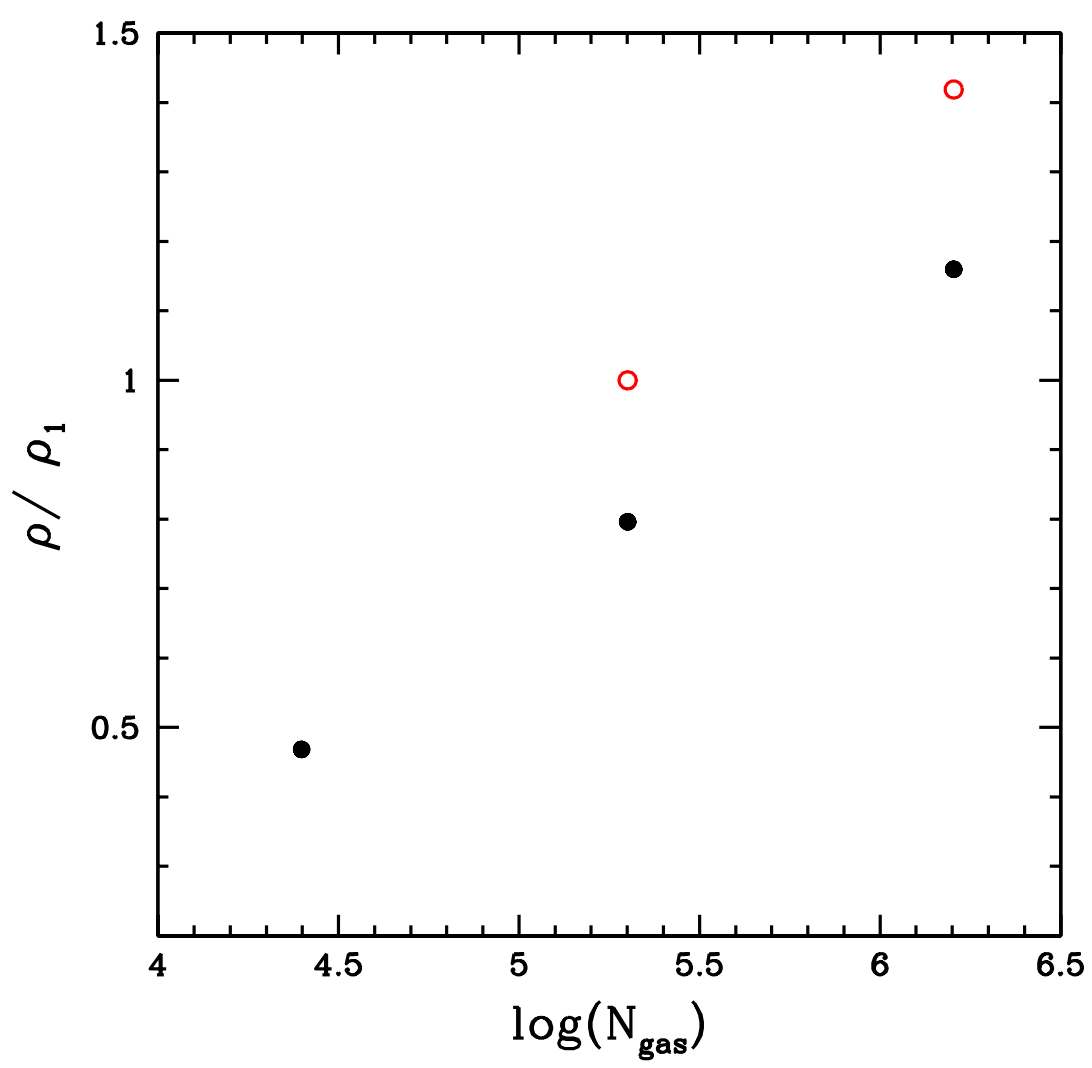

Figure 7. The maximum density of the gas for runs with different mass and force resolution. For all the runs the density is measured after bar formation has triggered the gas inflow and its value relative to that in the run employed in the Letter is shown $\left(\rho_{1}=2 \times 10^{-23} \mathrm{~g} / \mathrm{cm}^{3}\right)$. Red open circles refer to runs in which the gravitational softening is equal to the run discussed in the Letter (35 pc), while black filled circles show the results for runs in which the softening was increased by a factor of 3 . Therefore, while red and black circles represent the results of two sequences of runs with varying mass resolution, comparing red and black circles at fixed number of particles shows the effect of changing the gravitational softening.

or by laminar viscous stripping $26,37,55,56,57$. As we have also shown elsewhere ${ }^{26}$, KelvinHelmoltz instabilities should not occur for dwarfs embedded in a massive dark halo because of the stabilizing effect of the gravity of the halo. This is the case even here. Following existing analytical predictions ${ }^{55}$ we estimate that the critical value of the gravity (in simulation units) to stabilize our gas disk against Kelvin-Helmoltz is $g_{c r} \sim 0.1$ initially, where $g_{c r}=2 \pi V^{2} / D R_{g}\left(V \sim 200 \mathrm{~km} / \mathrm{s}\right.$ is the average orbital velocity of the galaxy, $R_{g}=0.5$ $\mathrm{kpc}$ is the scale length of the gas component, and $D=\rho_{g} / \rho_{g h}$ is the ratio between the density of the gaseous disk at $R \sim R_{g}$ and the time averaged density of gaseous halo along the orbit of the galaxy). The self-gravity of the dwarf galaxy within $R_{g}$, which is mainly provided by the dark halo, is instead $g \sim 2.5>g_{c r}$, where $g=G M_{\text {halo }} / R_{g}{ }^{2}$. We note that $g>g_{c r}$ holds down to about $1 / 5$ of the disk scale length, and also for $R>R_{g}$. Hence the gaseous disk of the dwarf is initially stable to Kelvin-Helmoltz instabilities. 
When $g>g_{c r}$ the gas can still be stripped by laminar viscous stripping. The mass loss rate in this case is given by $\dot{M} \sim 12 / 2.8 \pi R_{g}{ }^{2} V\left(\lambda_{\max } / R_{g}\right)\left(c_{1} / V\right)^{55,57}$, where $\lambda_{\max }$ is the longest wavelength not stabilized by gravity. Application of this formula to our dwarf galaxy in motion through the hot galactic halo yields a characteristic timescale for laminar viscous stripping which is longer than the Hubble time, mostly because $\lambda_{\max }<R_{g}$. However, close to the second pericenter passage, the situation changes (see Figure 8). The density of the halo within $0.5 \mathrm{kpc}$ decreases by a factor of 4 as a result of the tidal shocks, so that $g$ drops to $\sim 0.6$ at one disk scale length, now very close to $g_{c r}$ (the latter has also decreased in the meantime, $g_{c r} \sim 0.3$, since the mean density of the gas has dropped). The gas component of the dwarf is now only marginally stable to Kelvin-Helmoltz instabilities, but using the formula to calculate the mass loss rate due to Kelvin-Helmoltz ${ }^{55}$ for $R_{g}=0.5 \mathrm{kpc}$ one derives a characteristic stripping timescale still quite long, i.e. comparable to the Hubble time. Indeed, in the simulations we do not see Kelvin-Helmoltz instabilities developing before the gas is removed. Instead, assuming that the longest unstable wavelength is $\lambda_{\max } \sim 0.5 \mathrm{kpc}$ one obtains a laminar viscous timescale of $\sim 2$ Gyr. The gas is indeed completely stripped $\sim 1$ Gyr later (it is possible that stripping is slightly enhanced by artificial viscosity of $\left.\mathrm{SPH}^{26}\right)$.

When the UV background is absent $g>>g_{c r}$ even at the second pericenter passage because (1) the density of the halo within $0.5 \mathrm{kpc}$ decreases by only $50 \%$ as the cold, concentrated gas reduces tidal heating (see section 5.1 above), and (2) $g_{c r}$ is smaller because the gas is colder and denser relative to the case where the UV radiation background is included, thereby increasing the value of $D$ (see above) by a factor of 3 . As a result, the system is very stable to disruption by Kelvin-Helmoltz instabilities. Likewise, the longest unstable wavelength is much smaller than the radius of the residual gas, which implies a laminar viscous timescale close to the Hubble time, similar to what we have obtained for the initial conditions. This is consistent with our result that the gas is not completely stripped when the UV background is absent. In conclusion, despite the strongly time-dependent behavior of all the variables involved in the problem, the results of the simulations are consistent with the expected characteristic timescales and relative importance of the various ram pressure stripping modes.

\subsection{Adding complexity to the scenario}

The "proximity" effect due to the local ionizing background from the Milky Way at $z>1$, not included here, could have been even more important than that of the uniform cosmic UV background. Galaxy formation simulations that include star formation and the effect of supernova feedback show that star formation rates comparable to those of a luminous infrared galaxy $(\mathrm{LIRG}),>10 M_{\odot} / \mathrm{yr}$, are attained around $z \sim 2$ for a Milky Way-type galaxy $^{16}$. We have run a test with the addition of the local ionizing radiation produced by the starburst, found complete ram pressure stripping already on the first orbit and, as a consequence, an even faster transformation of the stellar component. 


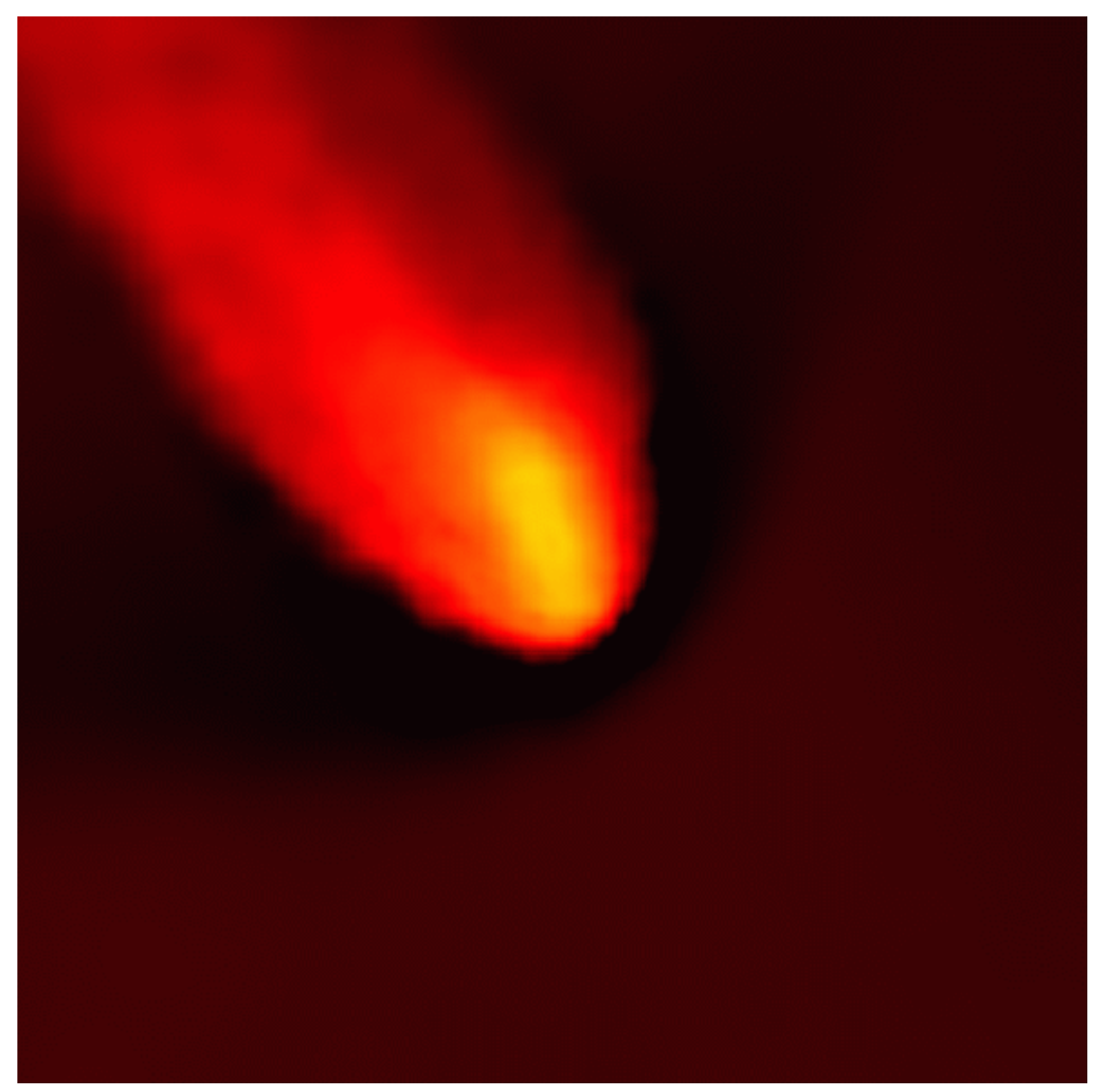

Figure 8. Color-coded projected density map of the gas component. The box is $3 \mathrm{kpc}$ on a side and shows the gas distribution view from above the orbital plane. The time is close to the second pericenter passage, after about 3 Gyr of evolution. The box corresponds to a zoom in of the middle right panel in Figure 1 of the Letter, the density limits are also similar, from $10^{-31}$ $\mathrm{g} / \mathrm{cm}^{3}$ (black) to $10^{-23} \mathrm{~g} / \mathrm{cm}^{3}$ (yellow).

The processes discussed in this paper assume that dSphs are bound satellites of a primary galaxy. Isolated $\mathrm{dSphs}$ such as Tucana are found at distances of order twice the expected virial radius of the Milky Way or M31 ${ }^{47,50}$. Either they are not bound to either of the primary galaxies or are extreme satellites on nearly radial orbits (in cosmological simulations a few satellites whose orbital apocenter greatly exceeds the virial radius are typically found $\left.{ }^{18,47}\right)$. In either case they cannot be objects that fell early into the halo of a primary galaxy (if bound, their orbital time would be too long to be consistent with an object infalling at $z>1$ ). Instead, their mass-to-light ratios are likely dictated by their halo mass rather than by the influence of the environment. Either their halos have a relatively small mass, $V_{\text {peak }}<20 \mathrm{~km} / \mathrm{s}$, so that reionization alone could have been sufficient at suppressing their baryonic content and star formation by inhibiting the collapse of gas and/or photoevaporating already accreted gas $^{53}$, or their halos have masses comparable to those of Draco but their mass-to-light ratios are much lower, comparable to those of 
Mayer et al.

the other dSphs, because baryonic sweeping was not effective. Transformation from disklike progenitors into dSphs could still be the origin of the diffuse spheroidal structure of Tucana since tidal stirring can occur even outside the virial radius of the primary galaxies in cold dark matter models, for example between members of subgroups infalling into the primaries $^{58}$. Improving our knowledge on the structure and properties of isolated dSphs can thus yield important constraints on formation scenarios such as that proposed in this Letter. Obtaining internal velocities to measure their halo mass and determine their massto-light ratio as well as achieving accurate upper limits on their gas content should be priorities among the new observational programs of nearby dwarfs.

Finally, we have only considered NFW profiles for the halos of the dwarfs. Recent work $^{45}$ has shown that a cuspy dark matter halo fitted by an NFW or Moore profile might develop a core as a result of a strong mass outflow such as that caused by supernovae winds after a burst of star formation. Our scenario for baryonic sweeping would be even stronger if dSphs had cored halos. Indeed, as we have seen in the previous section, the various modes of ram pressure stripping are all enhanced if self-gravity is lower, as it would be the case for a cored halo as opposed to a cuspy halo. Stripping will be much faster especially if the size of the core is comparable to the disk scale length since, as we have explained in section 6.1, the inner region is that in which instantaneous stripping is not effective and other slower stripping regimes such as viscous or turbulent stripping come into play. Tidal shocks will also be enhanced because the response of the system will be more impulsive, an effect which is already seen when comparing subhalos with NFW profiles having different concentration parameters ${ }^{59}$. On the other hand, dwarf galaxies having the low gas surface densities and high Toomre parameter of our initial model would hardly undergo a strong burst but rather have a fairly low star formation rate ${ }^{35}$, in which case modifications of the halo profile are unlikely.

Received; Accepted .

1. Wadsley, J., Stadel, J., \& Quinn, T., Gasoline: a flexible, parallel implementation of TreeSPH. New Astr. 9, 137-158 (2004)

2. Barnes, J., \& Hut, P., A Hierarchical $\mathrm{O}(\mathrm{N} \log \mathrm{N})$ Force-Calculation Algorithm. Nature 324, 446-449 (1986)

3. Hernquist, L., Bouchet, F., and Suto, Y., Application of the Ewald method to cosmological N-body simulations. Astrophys. J. Supp 75, 231-240 (1991)

4. Gingold, R.A. \& Monaghan, J.J. Smoothed particle hydrodynamics: theory and application to non-spherical stars. Mon. Not. R. Astron. Soc. 181, 375-389 (1977)

5. Monaghan, J.J., Smoothed particle hydrodynamics. Annual. Rev. Astron, Astrophys. 30, 543$574(1992)$

6. Springel, V., \& Hernquist, L. Cosmological smoothed particle hydrodynamics simulations: the entropy equation. Mon. Not. R. Astron. Soc. 333, 649-664 (2002) 
7. Haardt , F. \& Madau. P., Radiative Transfer in a Clumpy Universe. II. The Ultraviolet Extragalactic Background. Astrophys. J. 461, 20-37(1996)

8. Alvarez, M.A., Shapiro, P., Kyungjin A. \& Iliev, I.T. Implications of WMAP 3 Year Data for Sources of Reionization. Astrophys. J. 644 L101-L104 (2006)

9. Grebel, E.K., Gallagher, J.S., \& Herbeck, D. The Progenitors of Dwarf Spheroidal Galaxies. Astron. J. 125, 1926-1939 (2003)

10. Balsara, D.S., von Neumann stability analysis of smoothed particle hydrodynamics:suggestions for optimal algorithms. J.Comput. Phys. 121 357-372 (1995)

11. Governato, F., Mayer, L., Wadsley, J., Gardner, J. P., Willman, Beth, Hayashi, E., Quinn, T., Stadel, J.\& Lake, G, The Formation of a Realistic Disk Galaxy in Lambda-dominated Cosmologies. Astrophys. J. 607, 688 (2004)

12. Klypin A., Zhao H., Somerville R. S., $\Lambda$ CDM-based Models for the Milky Way and M31. I. Dynamical Models, Astrophys. J., 573, 597-613 (2002)

13. Efstathiou, G., et al.,Evidence for a non-zero Lambda and a low matter density from a combined analysis of the $2 \mathrm{dF}$ Galaxy Redshift Survey and cosmic microwave background anisotropies. Mon. Not. R. Astron. Soc. 330, L29-L35 (2002)

14. Power, C., et al.,The inner structure of LambdaCDM haloes - I. A numerical convergence study. Mon. Not. R. Astron. Soc. 338, 14-34 (2003)

15. Gardner J. P., Dependence of Halo Properties on Interaction History, Environment, and Cosmology, Astrophys. J., 557, 616-625 (2001)

16. Governato et al. Mon. Not. R. Astron. Soc. in press

17. Katz, N., Quinn, T., Bertschinger, E., \& Gelb, J.M., Formation of Quasars at High Redshift, Mon. Not. R. Astron. Soc. 270, L71-L74 (1994)

18. Ghigna, S., Moore, B., Governato, F., Lake, G., Quinn, T., \& Stadel, J., Dark matter haloes within clusters, Mon. Not. R. Astron. Soc. 300, 146-162 (1998)

19. Moore, B., et al., Cold collapse and the core catastrophe, Mon. Not. R. astron. Soc. 310, 1147-1152 (1999)

20. Mastropietro, C., Moore, B., Mayer, L., Debattista, V., Piffaretti, R., \& Stadel, J., Morphological evolution of discs in clusters. Mon. Not. R. astron. Soc. 364, 607-619 (2005)

21. Hernquist, L., N-body realizations of compound galaxies, Astrophys. J. Supp. 86, 389-400 (1993)

22. Springel, V. \& White, S.D.M.,Tidal tails in cold dark matter cosmologies. Mon. Not. R. Astron. Soc. 307, 162-178 (1999)

23. Fall S. M., \& Efstathiou G., Formation and rotation of disc galaxies with haloes, Mon. Not. R. Astron. Soc. 193, 189-206 (1980) 
24. Mo, H. J., Mao \& White, S. D. M. , The formation of galactic discs, Mon. Not. R. Astron. Soc. 295, 319-336 (1998)

25. Mayer, L., Moore, B., Quinn, T., Governato, F., \& Stadel, J., Tidal debris of dwarf spheroidals as a probe of structure formation models, Mon. Not. R. Astron. Soc. 336, 119-130 (2002)

26. Mayer, L., Mastropietro, C., Wadsley, J., Stadel, J., \& Moore, B., Simultaneous ram pressure and tidal stripping; how dwarf spheroidals lost their gas, Mon. Not. R. Astron. Soc. 369, 10211038 (2006)

27. Colin, P., Klypin, A., Valenzuela, O., \& Gottloeber, S., Dwarf Dark Matter Halos. Astrophys. J. 612, 50-57 (2004)

28. Navarro, J. F., Frenk, C. S., White, S. D. M., The Structure of Cold Dark Matter Halos, Astrophys. J. 462, 563-575 (1996)

29. Jimenez, R., Verde, L., \& Oh, S. P., Dark halo properties from rotation curves, Mon. Not. R. Astron. Soc. 339, 243-259 (2003)

30. Mayer, L., \& Moore, B, The baryonic mass-velocity relation: clues to feedback processes during structure formation and the cosmic baryon inventory, Mon. Not. R. Astron. Soc. 354, 477-484 (2004)

31. Verde, L., Oh, S.P., \& Jimenez, R., The abundance of dark galaxies, Mon. Not. R. Astron. Soc. 336, 541-549 (2002)

32. Wong. T., \& Blitz, L., 2002, The Relationship between Gas Content and Star Formation in Molecule-rich Spiral Galaxies, Astrophys. J. 569, 157-183 (2002)

33. Vallenari, A., Schmidtobreick, L., \& Bomans, D.J., The star formation history of the LSB galaxy UGC 5889, Astr. and Astrophys. 435, 821-829 (2005)

34. Schaye, J., Star Formation Thresholds and Galaxy Edges: Why and Where. Astrophys. J. 609, 667-682 (2004)

35. Li, Y., Mac Low, M., \& Klessen, R., Star Formation in Isolated Disk Galaxies. I. Models and Characteristics of Nonlinear Gravitational Collapse, Astrophys.J. 626, 823-843, (2005)

36. Klein, R., McKee, C.F., \& Colella, P., On the hydrodynamic interaction of shock waves with interstellar clouds. 1: Nonradiative shocks in small clouds, Astrophys. J. 420, 213-236 (1994)

37. Mac Low, M, \& Zahnle, K., Explosion of comet Shoemaker-Levy 9 on entry into the Jovian atmosphere. Astrophys. J. 434, L33-L36 (1994)

38. Bate, M., \& Burkert, A., Resolution requirements for smoothed particle hydrodynamics calculations with self-gravity, Mon. Not. R. Astron. Soc. 288, 1060-1072 (1997)

39. Dehnen, W., \& Binney, J., Mass models of the Milky Way, Mon. Not. R. Astron. Soc. 294, 429-434 (1998)

40. Lin, D.N.C., Jones, B.F., \& Klemola, A.R., The motion of the Magellanic clouds, origin of the Magellanic Stream, and the mass of the Milky Way Astrophys. J. 439, 652-671 (1995) 
41. Sembach, K.R., et al., Highly Ionized High-Velocity Gas in the Vicinity of the Galaxy, Astrophys. J. Supp. 146, 165-208 (2003)

42. Mastropietro, C., Moore, B, Mayer, L., Wadsley, J., \& Stadel, J., The gravitational and hydrodynamical interaction between the Large Magellanic Cloud and the Galaxy, Mon. Not. R. Astron. Soc., 363, 509-520 (2006)

43. Navarro, J. F., Eke, V.R., \& Frenk, C.S., The cores of dwarf galaxy haloes, Mon. Not. R. Astron. Soc. 283, L72-L78 (1996)

44. Gnedin, O., \& Zhao, H., Maximum feedback and dark matter profiles of dwarf galaxies Mon. Not. R. Astron. Soc. 333, 299-306 (2002)

45. Read, J, \& Gilmore, G., Mass loss from dwarf spheroidal galaxies: the origins of shallow dark matter cores and exponential surface brightness profiles, Mon. Not. R. Astron. Soc. 356, 107-124 (2005)

46. Dubinski, J., \& Carlberg, R.G., The structure of cold dark matter halos, Astrophys. J. 378, 496-503 (1991)

47. Mateo, M., 1998, Dwarf Galaxies of the Local Group, Annual Review of Astronomy and Astrophysics 36, 435-506 (1998)

48. James, P., An infrared study of dwarf galaxies in the Virgo cluster, Mon. Not. R. Astron. Soc. 250, 544-554 (1991)

49. Mayer, L., Governato, F., Colpi, M., Moore, B., Quinn, T., Wadsley, J., Stadel, J., Lake G., Tidal Stirring and the Origin of Dwarf Spheroidals in the Local Group, Astrophys. J. 547, L123-L127 (2001)

50. Mayer, L., Governato, F., Colpi, M., Moore, B., Quinn, T., Wadsley, J., Stadel, J., \& Lake, G., The Metamorphosis of Tidally Stirred Dwarf Galaxies, Astrophys. J. 559, 754-784 (2001)

51. Hargreaves, J. C., Gilmore, G, Irwin, M. J.\& Carter, D., A Dynamical Study of the UrsaMinor Dwarf Spheroidal Galaxy, Mon. Not. R. Astron. Soc. 271, 693-705 (1994)

52. Armandroff, T. E., Olszewski, E. W, Pryor, C., The Mass-To-Light Ratios of the Draco and Ursa Minor Dwarf Spheroidal Galaxies.I. Radial Velocities from Multifiber Spectroscopy, Astron. J. 110, 2131-2165 (1995)

53. Susa, H., \& Umemura, M., Formation of Dwarf Galaxies during the Cosmic Reionization, Astrophys. J. 600, 1-16 (2004)

54. Gunn, J. E., Gott, J. R. I., On the Infall of Matter Into Clusters of Galaxies and Some Effects on Their Evolution, Astrophys. J 176, 1-19 (1972)

55. Murray, S. D., White, S. D. M., Blondin, J. M., \& Lin, D. N. C., Dynamical instabilities in two-phase media and the minimum masses of stellar systems, Astrophys. J. 407, 588-596 (1993)

56. Quilis, V., Moore, B \& Bower, R., Gone with the Wind: The Origin of S0 Galaxies in Clusters, Science. 288, 1617-1620 (2000) 


\section{Mayer et al.}

57. Nulsen, P. E. J., Transport processes and the stripping of cluster galaxies, Mon. Not. R. Astron. Soc. 198, 1007-1016 (1982)

58. Kravtsov, A. V., Gnedin, O. Y., Klypin, A. A. The Tumultuous Lives of Galactic Dwarfs and the Missing Satellites Problem, Astrophys. J , 609, 482-497 (2004)

59. Kazantzidis, S., Mayer, L., Mastropietro, C., Diemand, J., Stadel, J., \& Moore, B., Density profiles of Cold Dark Matter substructure: implications for the missing-satellites problem, Astrophys. J., 608, 663-679 (2004) 